\title{
DIGITAL STORYTELLING WITH DINAH: DYNAMIC, INTERACTIVE, NARRATIVE AUTHORING HEURISTIC
}

\author{
David Ventura ${ }^{1}$, David Brogan ${ }^{2}$ \\ 1 Entertainment Technology Center, Carnegie Mellon University \\ 2 Department of Computer Science, University of Virginia \\ \{dventura@cmu.edu\}
}

\begin{abstract}
We present a novel method for defining and assembling interactive narrative while operating under an optimized constraint-based system. DINAH is a dynamic, interactive, narrative authoring heuristic that constructs textual stories from a database of short story components. Similar to video game techniques that blend short motion capture segments to construct interactive graphical characters, DINAH builds entire narratives from a database of indexed story segments. The narrative DINAH builds dynamically adjusts to a user's interaction while run-time algorithms ensure that the essential ingredients of a compelling narrative are preserved. We use DINAH to further explore the potential and utility of interactive, computer-generated narrative, both as a learning tool and an entertainment technology.
\end{abstract}

Key words: interactive narrative, digital storytelling, optimized constraints

\section{Introduction}

Digital storytelling is a relatively new field that utilizes computers and other electronically augmented devices as a medium for the conveyance of stories. Digital storytelling may be used for a wide variety of purposes ranging from business communication and presentation, to personal expression, to interactive narrative. A narrative is interactive when the story contours to the user, based on his/her own personal preferences and input.

The original version of this chapter was revised: The copyright line was incorrect. This has been corrected. The Erratum to this chapter is available at DOI: 10.1007/978-0-387-35660-0_65 
Although an author could build an interactive narrative by writing multiple permutations of a single dramatic theme, such solutions do not scale to large stories with many user-directed forking points.

DINAH is an authoring tool used for the creation of interactive narrative. To use DINAH, the author fills a relational database with many small story clips, each one tagged with constraints that specify how it can be combined with others. For example, a story clip that describes the dramatic reunion of two brothers must include a precondition constraint that the user is aware that the two men were brothers and had been separated. After the clips are labeled, they are read into a database and assembled by the narrative engine at run time. A story may then be told to the user by DINAH. At certain points in the story, DINAH dynamically selects the most appropriate story clips and prompts the user to play the role of the avatar, thus building the story. This interaction loop gradually culls potential story clips from the database, further refining the story to suit the user's unique preferences. This process is repeated until the story is terminated when any one of many potential conclusions are reached.

\section{Related Work}

Digital storytelling is by nature interdisciplinary, and as such a basic understanding of both narrative composition in a traditional sense and the electronic format must be attained. Literature and film composition have long since been examined and so it is advantageous to ground the metric and constraints for an interactive system in this research.

\subsection{Traditional Narrative Structure Analysis}

Metalinear narrative is a special class where the narrative is broken up to include multiple pathways, each connected by a series of common elements or fragments. The literature of Borges best illustrates this principle. His Garden of Forking Paths suggests time as being comprised of a set of branching realities [2]. Borges' stories create a sense of multidimensional space that is generated from reading the linear text. In a more simplified and commercial manner, metalinear narrative also has its influences in the classic Choose Your Own Adventure books originated by Packard in the 1970s [9]. These books contain a finite number of plot lines and narrative paths, broken up by waypoints at critical moments in the story. At these moments, the reader is prompted to play the role of the characters and make a choice between several possible actions, directing him to another page. 
Early in the twentieth century, Propp constructed a set of narrative traits to aid in cataloging Russian fairy tales [11]. He made comparisons between the common elements of the narratives and produced thirty-one main types of stories, each with a number of variations. Upon encoding a value to each it was possible to describe any Russian folk tale using three qualifiers. More recently, Branigan composed a model of cinematic narrative structure, which builds on a well-defined system of constraints [3]. His model includes seven stages:

1. introduction of setting and characters

2. explanation of a state of affairs

3. initiating event

4. emotional response or statement of a goal by the protagonist

5. complicating emotions

6. outcome

7. reactions to outcome

Branigan's model, as it applies to western culture stories, is ideal for providing a metric under which interactive narrative may be evaluated. A story that satisfies Branigan's model must contain all seven stages, though repetition of some stages is permitted to increase the depth of causal relationships.

\subsection{Digital Narrative Composition}

Narrative structure has been analyzed extensively for the purposes of multimedia and education. Miller suggests that narrative can be built piecemeal by subnarratives [8]. These sub- or micronarratives are more likely not only to suit interaction (interactivity) but also to encourage it. Hughes describes narratives as three-dimensional spaces, or landscapes (as opposed to paths) [7]. He goes so far as to define narrative as possibly "everything," suggesting a "Particle Physics of narrative," with tiny narratives of "50 milliseconds to 3 seconds." This reinforces his belief that "big narrative emerges from little narrative," and Miller's theory of micronarrative. Hughes also suggests that narrative of high quality should not be written open-ended and generic.

In Brooks' Agent Stories suite, he provides an encompassing test bed for applying his model of metalinear narrative [5]. Agent Stories is a graphical toolkit for metalinear narrative creation. Story elements, or clips, are represented as graphical elements in an interactive application that permits authors to create associations and form a testbed for metalinear narrative interpretation. Our work is most closely related to Brooks' [4,5], as we suggest a method for metalinear story authorship, based on Branigan's model for narrative composition. 


\section{Interactive Narrative}

Many modern implementations of computer-generated narrative are built from large, preset blocks of text constructed by the author, with either a set path for the plot to follow, or a significant amount of pure randomization to prompt variance in the experience. These approaches require skillful craftsmanship by the author and explicit identification of numerous story paths to an interactive and immersive experience. DINAH seeks to provide interactive narrative dynamically by using narrative theories to continuously adapt to the user's interactions while preserving dramatic content. DINAH provides a dynamic run-time narrative, as opposed to a strict path that the user must follow to interact with the story.

The main challenge of building a system like DINAH is to preserve the story designer's dramatic vision while providing interactivity to the user. At one end of the spectrum we allow the users complete freedom to do whatever they please in their environment, without clear goals or limitations. This is much like online communities such as The Realm [6], where the users' goals consist of survival (or death if it interests them) and wandering the countryside while doing as they like to the environment and the other users. At the other end of the spectrum, we have the archetypical "good story": immersive, extremely detailed and well thought out. The reader is more or less constrained to experience the story exactly as the author intended, without deviation or variance. This is comparable to the epic film or engrossing book. DINAH hopes to straddle these two traditionally exclusive forms of narrative and provide an engaging hybrid.

\section{Implementation}

DINAH is composed of a story clip database that models the components of narratives and a set of algorithms that prescribe the combination of these components to build a story. The story clips are implemented as brief snippets of action or observation, with as little dependency on one another as possible. Each clip contains explicit pre- and postconditions that define limitations on when it can be added to the story and how the story is changed. A set of state vectors, called the story snapshot, is defined at runtime to permit fast verification of preconditions and simple accounting of postconditions. The algorithms that combine the clips form a narrative engine, which coordinates the concatenation of clips into a story that adheres to Branigan's model. In this system, we pay particular attention to the temporal relationships between story clips and define four dimensions of time that must be obeyed. 
Because DINAH takes a character-based view of narrative progression, both the avatar and all supporting characters are modeled with an elementary set of states, the character states. These states describe moods like happiness, boredom, and attraction as well as discrete relationships about where a player has been, whom the player has seen, and what additional special events have occurred. These variables influence how characters behave and thus help drive the narrative progression. The world itself possesses a state, the world state, that maintains omnipotent factors such as location, time, and the physical status of key inanimate objects.

This state-based representation of characters is used by developers of interactive characters $[1,10]$, but the DINAH story snapshot also includes a narrative state vector that encodes the progress towards completing a narrative (per Branigan's model). The character, world and narrative states are perhaps the most important constraints to ensuring that a good tale is generated. They embody the essentials of the entire narrative and control the flow of the story, ensuring all of the narrative elements are included for causality.

\subsection{Story Clips}

Story clips are encoded with a simple numerical ID tag, an abbreviated string for reference, text values, and the pre- and postconditions. The text values consist of a one-phrase summary of the user's possible action, and the corresponding world reaction. The preconditions are modeled as state vectors that describe the conditions of the characters, world, and narrative that must be observed before the clip can be executed. The comparison of the preconditions to the state of the story is accomplished via the story snapshot, which economically and quantitatively represents the three important state vectors. Similarly, the postconditions define changes to the character, world, and narrative states vectors of the story snapshot.

\subsection{The Narrative Engine}

The narrative engine is the core for production of interactive stories (figure 1). The run-time character, world, and narrative state vectors are compared to the precondition constraints of the clips stored in the database to extract the most appropriate story clip candidates. This comparison is accomplished by computing the vector difference between each story clip's preconditions and the current story snapshot. The difference vector is scaled by a proportional weighting vector to emphasize particular state vector components. The candidate clips specify potentially divergent plot options and some differences may rule out a clip completely, such as narrative and 
temporal cohesion. But variance is allowed and expected in the character states, as they describe one moment of time where each of the individuals in the story had one of many emotional combinations. The narrative engine orders the clips according to the quality of their match with the current story state.

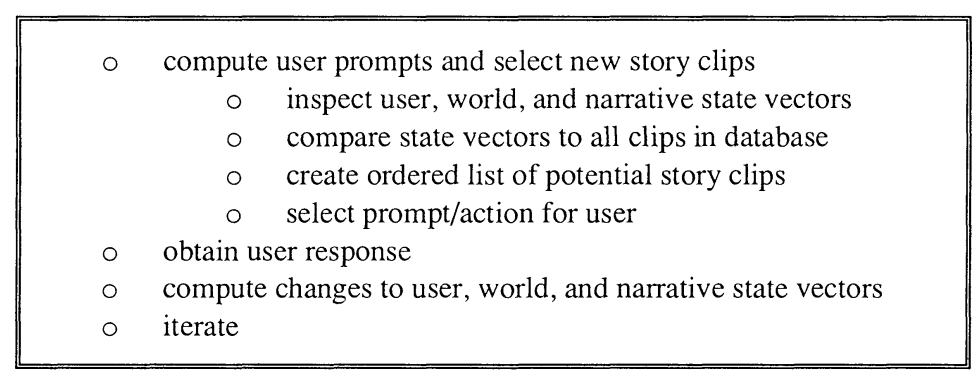

Figure 1. DINAH storytelling engine main algorithm.

The narrative engine uses the evaluated story clips to present the user with the opportunity to choose the branching of the story while constraining the story divergence in a feasible manner. When multiple story clips are viable at one time (which occurs quite frequently), the narrative engine queries the user to select one clip from a list of the four most similar to the current story state, presenting the most closely matching option first. The textual query presented to the user is constructed from the textual summaries associated with each of the four similar clips. If fewer than four possible clips exist, all the feasible alternatives are presented. If only one clip is appropriate, it is issued to the user automatically, providing the text connecting the previous and following causal events. The user's selection is integrated into the story, and the clip's postconditions are used to update the current story's state vectors, recording the narrative's progress.

The user queries are functionally prompts for possible paths of action, but they are presented in a more introspective manner. The user's task at story branch points consists of completing a sentence describing how the avatar currently feels, either about a particular character or the situation in general. These predicates are in actuality the contending clip summaries selected by the engine which are linked to physical events such as discussing a certain topic with a character or performing some sort of action on one of the world's inanimate objects. This presentation gives an added sense of appeal and charm by enticing the user to play the role of the avatar in a more intellectual sense than a more traditional, omnipotent physical one. 


\subsection{Temporal Coherence}

The passage of time and the dependencies it places on events is one of the greatest constraints in creating narrative. The story needs to be flexible enough that the user has a sense of freedom and control of the environment, but methodical and ordered so the triggering of events makes sense and a satisfying depth of causality can be obtained. To help enforce these limitations, the DINAH engine uses a four-dimensional model of time to cull inappropriate clips.

A clip that satisfies Branigan's narrative model is submitted to a series of time comparisons to verify that the prospective clip may follow the current story state. The narrative engine performs these temporal evaluations by checking the potential clip against four dimensions: Major Sequential Time (MaST), Delta Time (DT), Mutually Exclusive Time (MET), and Minor Sequential Time (MiST) (figure 2). MaST denotes the passage of superphases in one's life. DT allows for the execution of any subsets of narrative, however activities from the sets may not overlap in time, like a temporary form of mutual exclusion. MET culls branches that must be forever mutually exclusive, as the occurrence of one precludes the other. MiST controls the micronarrative order on an event-by-event basis.

1. You must complete high school before completing college (MaST).

2. You may get a degree from Carnegie Mellon, and you may get a degree from Virginia, but never at the same time (DT).

3. You may marry a girl and be wed, or not and stay a bachelor, but once you marry, you can no longer be a bachelor (MET).

4. You must complete your college application before you can mail it (MiST).

Figure 2. Four dimensions of time as an adolescent.

By monitoring the flow of time through the four dimensions, the user is allowed to roam freely through certain areas and perform any variety of activities provided, regardless of whether they may or may not have a grand impact on the narrative. When a major progression does occur, however, the engine promotes it and removes any now unrelated or inappropriate events or actions, allowing a new set of possible clips to affect the story. In each story clip the four dimensions of time are encoded as a series of ascending integers, greater numbers indicating later potential occurrence. 


\section{Preliminary Experiments}

Friday Night is the sample set of story components for the first DINAH prototype. The story begins when the user's avatar arrives at a bar late Friday evening. The clip database contains the adventures the avatar encounters after he enters the tavern. There are nine possible endings to the story, all entirely different. The paths to reach these endings are many and varied. The database of story clips is richer in breadth than depth for this first iteration, with a total of about 50 story clips all together. Clips are defined as being either a termination (ending), prompt (choice), or reaction (intermediate result).

In performance testing, a simulated user produced a sequence of 50 random inputs in an attempt to break the system. In all the tests, the program responded intelligently without becoming ensnared in any recursive or nonterminating narrative loops. Also, causality and temporal coherence were always maintained, as the responses that were issued were always valid within the context of the given story.

After technical proving, the system was tested on a dozen college students from engineering and humanities backgrounds. Responses were encouraging, as the majority of the users were engaged with the story and exhibited positive reactions to the experience. The users were asked to describe the most positive and negative elements of the interaction. The most positive comments made cite the narrative as lush, descriptive, and that it presented appealing conflicts. The negative criticisms received were for a better user interface to the stories and a sense of greater freedom for the avatar. These findings reinforced that a rich presentation is critical and the content has to be personally identifiable. Also, the struggle between being guided yet unhindered was also reaffirmed.

\section{Conclusions and Future Work}

One of the most valuable lessons learned in producing DINAH was how challenging it is to maintain a strong sense of history in the stories. This makes a good argument for discrete-event (implicit) narrative. In implicit narrative, events themselves generate stories, which arguably may be overly uninteresting, but the self-motivated activity of creating story from a series of character behaviors can be greatly enjoyable, as is witnessed in many user fan pages of the computer game The Sims [12]. In one prototype, strict rules of narrative and time enforcement were abandoned and the characters' state was the sole fuel for the engine. This emotional model is much more liberal, prompting a wider range of responses and freedom for the user. However, it 
is more difficult for the characters to react intelligently to the user, and so there is the drawback of a demand for believable AI.

A marked characteristic of emotion-driven stories is a sense of "dead time," where it is sometimes best for the story if nothing happens. With the final prototype we developed, we brought the two aspects of computer-told stories together: a strong governing force to ensure story progress to an ultimate end and the freedom and flexibility to not hinder users to narrow range of sequences. Overall, we were pleased with the performance and quality of the stories generated by DINAH, especially because the clips needed little modification after their initial encoding.

In the future, the DINAH narrative engine may progress to be flexible enough that it will learn the genre of story components it is assembling, and thusly react in an appropriate manner. There are several questions that spring to mind considering the scope of this project. Can DINAH integrate storytelling into a structured education environment? How large a role could an engine similar to DINAH play in creating unique experiences for game players? The potential of the system is quite intriguing and invites further examination.

\section{References}

[1] Blumberg, Bruce. Old Tricks, New Dogs: Ethology and Interactive Creatures. MIT PhD Dissertataion, 1996.

[2] Borges, Jorge Luis. Labyrinths. New York, New York: Modern Library, 1983, c1964.

[3] Branigan, Edward. Narrative Comprehension and Film. New York, New York: Routledge. 1992.

[4] Brooks, Kevin M. Do Agent Stories Use Rocking Chairs: The Theory and Implementation of One Model for Computational Narrative. ACM Multimedia '96. 1996.

[5] Brooks, Kevin M. Metalinear Cinematic Narrative: Theory, Process, and Tool. Ph.D. Dissertation, Massachusetts Institute of Technology, School of Architecture and Planning. April 1999.

[6] Codemasters Software Company Limited. The Realm Online. http://www.realmserver.com. 2001.

[7] Hughes, Bob. Narrative as Landscape. Proceedings of Narrative and Hypermedia Workshop, Multimedia, Education, and Narrative Organisation. 1997.

[8] Miller, Hugh. Micronarrative and Interaction. Proceedings of Narrative and Hypermedia Workshop, Multimedia, Education, and Narrative Organisation. 1997.

[9] Packard, Edward. Choose Your Own Adventure Series. Random House. 1979.

[10] Perlin, Ken and Goldberg, Athomas. Improv: A System for Scripting Interactive Actors in Virtual Worlds. Proceedings of SIGGRAPH '96, p 205-216.

[11 ] Propp, Vladmir. Morphology of the Folktale (Laurence Scott, Trans.). Austin, Texas: University of Texas Press. 1968.

[12] Wright, Wil. The Sims. Maxis/EA. 2000. 